\title{
Interpolation Theory and the Wigner-Yanase-Dyson-Lieb Concavity
}

\author{
Hideki Kosaki` \\ Department of Mathematics, The University of Kansas, Lawrence, KS 66045 USA
}

\begin{abstract}
The Wigner-Yanase-Dyson-Lieb concavity is naturally captured in the frame of interpolation theory. Among other results, a certain generalization (involving operator monotone functions) of this concavity in the context of general von Neumann algebras is obtained. Also, a close relationship between the above subjects and F. Hansen's inequality is clarified. All results are proved by using simple variational expressions of involved quantities.
\end{abstract}

\section{Introduction}

Since the joint concavity of $\operatorname{Tr}\left(a^{1-\theta} x^{*} b^{\theta} x\right), 0 \leqq \theta \leqq 1$, in $(a, b)$ was proved by Lieb, [22], it is referred to as the Wigner-Yanase-Dyson-Lieb (WYDL) concavity. Here, $a$ and $b$ (respectively $x$ ) are positive (respectively an arbitrary) matrices. Then, Araki, [6], obtained the corresponding result for general von Neumann algebras. Due to the fact that this concavity has important applications to theoretical physics and information theory (subadditivity for entropy, etc., see Sect. 8, [28], for example), several authors have been trying to obtain various generalizations in many directions, [11, 24, 25, 30].

Proofs in $[6,22]$ are based on the Phragmen-Lindelöf theorem (complex interpolation). The purpose of the article is to capture the WYDL concavity in the frame of general interpolation theory. Especially, we examine a certain real interpolation method (the $K$-method of Peetre) in Sect. 1, and quadratic interpolation methods in Sects. 2 and 3. Also, as applications of our arguments, in Sect. 4 we establish a close relation among this subject, F. Hansen's inequality [15], and operator monotone functions [10], while in Sect. 5 we obtain a certain generalization of Araki's version, [6], of the WYDL concavity in the context of general von Neumann algebras.

We show that, in our frame, the WYDL concavity is a natural and common phenomenon (Theorems $1.8,3.5$ ) due to the fact that many involved quantities

\footnotetext{
^Supported in part by the National Science Foundation, grant number MCS- 8102158

Current Address: Department of Mathematics, Purdue University, West Lafayette, IN, 47907 USA
} 
(interpolation norms) admit variational expressions. This key fact follows from our main technical lemma (Lemma 2.1).

\section{Real Interpolation Method (the K-Method of Peetre)}

We recall the $K$-method of Peetre (one of real interpolation methods) as well as the general interpolation theory. Our basic reference is [8].

Throughout the paper, let $V$ be a vector space equipped with two norms \|\|$_{0}$, \| $\|_{1}$ which are compatible (see Remark 2.3) in the following sense: Any sequence in $V$ which converges to 0 in one norm and is Cauchy in the other norm must converge to 0 in the other norm. Under these circumstances, the completions of $V$ under the norms \|\|$_{0},\|\quad\|_{1}$ are continuously imbedded into the completion of $V$ under the norm

$$
\|v\|=\inf \left\{\left\|v_{0}\right\|_{0}+\left\|v_{1}\right\|_{1} ; \quad v=v_{0}+v_{1}\right\} .
$$

(See p. 35, [26], for example.) Hence, one obtains a compatible couple of Banach spaces in the sense of [8]. (See [7] for more detailed study of compatibility.) However, we do not consider these "redundant" completions usually (except in Sect. 3), and will deal with a triple $\left(V,\|\|_{0},\|\|_{1}\right)$.

To explain what an interpolation method is, we consider one more generic $\left(W,\|\|_{0, W},\|\|_{1, W}\right)$ and an arbitrary linear operator $T$ from $V$ into $W$ satisfying

$$
\left.\begin{array}{ll}
\|T v\|_{0, W} \leqq M_{0}\|v\|_{0}, & v \in V, \\
\|T v\|_{1, W} \leqq M_{1}\|v\|_{1}, & v \in V .
\end{array}\right\}
$$

A method $F$ of constructing a new norm \|\|$_{*}=F\left(\|\|_{0},\|\|_{1}\right)$ on $V$ (hence also - \|\|$_{*, W}=F\left(\|\quad\|_{0, W},\|\quad\|_{1, W}\right)$ on $\left.W\right)$ satisfying

$$
\|T v\|_{*, W} \leqq M_{*}\|v\|_{*}, \quad v \in V
$$

is the main concern of interpolation theory. If (2) always implies (3) for any $\left(V,\|\|_{0}\right.$, \|\|$\left._{1}\right),\left(W,\|\|_{0, W},\|\|_{1, W}\right)$, and $T$, then a method $F$ is called an interpolation method and the resulting norm \|\|$_{*}=F\left(\|\|_{0},\|\|_{1}\right)$ is called the interpolation norm (constructed from \|\|$_{0},\|\|_{1}$ by $F$ ). Many interpolation methods are known. (The complex method, the $K$-method, the $J$-method, etc., see [8].)

The reader familiar with general interpolation theory may have noticed that our definition above is quite restricted. However, for our purpose (WYDL concavity) the above definition is more convenient.

Definition 1.1. If (2) implies (3) with $M_{*}=M_{0}^{1-\theta} M_{1}^{\theta}, 0<\theta<1$, (respectively $\left.M_{*}=\operatorname{Max}\left(M_{0}, M_{1}\right)\right)$, then $F$ is said exact of exponent $\theta$ (respectively exact).

We now recall the $K$-method of Peetre which is one of real interpolation methods and is exact of exponent $\theta$.

Definition 1.2. ( $K$-functional) For each $t>0$, we set

$$
K(t, v)=\inf \left\{\left\|v_{0}\right\|_{0}+t\left\|v_{1}\right\|_{1} ; \quad v=v_{0}+v_{1}\right\}, \quad v \in V .
$$


More generally, for each $t>0$ and $p>0$, we set

$$
K_{p}(t, v)=\inf \left\{\left(\left\|v_{0}\right\|_{0}^{p}+t^{p}\left\|v_{1}\right\|_{1}^{p}\right)^{1 / p} ; \quad v=v_{0}+v_{1}\right\} .
$$

Then all norms $K(t, \cdot), t>0$, on $V$ are equivalent to the norm $(1)(=K(1, \cdot))$. We "superpose" these norms in the following way:

Definition 1.3. (The $K$-method of Peetre) For each $0<\theta<1$ and $1 \leqq q \leqq \infty$, we set

$$
\|v\|_{\theta, q}=\left[\int_{0}^{\infty}\left\{t^{-\theta} K(t, v)\right\}^{q} d t / t\right]^{1 / q}, \quad v \in V .
$$

(It is easily shown that $\|v\|_{\theta, q}<\infty$, see p. 40, [8].) This method of constructing \|\|$_{\theta, q}$ from a generic $\left(V,\|\|_{0},\|\|_{1}\right)$ is called the $K$-method (determined by $\theta, q)$.

The above described (usual) $K$-method is useful to deal with $L^{p}$-spaces and Lorentz spaces. However, we will need its variant, which will be described below.

Definition 1.4. For each $p>0$, we set

$$
\|v\|_{\theta, q: p}=\left[\int_{0}^{\infty}\left\{t^{-\theta} K_{p}(t, v)\right\}^{q} d t / t\right]^{1 / q}, \quad v \in V .
$$

Holmstedt and Peetre, [17], showed:

Proposition 1.5. For each $p>0$ (and $0<\theta<1,1 \leqq q \leqq \infty)$, the norm $\|\quad\|_{\theta, q ; p}$ is equivalent to the norm \|\|$_{\theta, q}\left(=\|\|_{\theta, q ; 1}\right)$. (In particular, \|\|$_{\theta, q ; p}$ is also an interpolation norm.)

The next result is well-known. However, we present its proof because it is intuitive to understand the nature of "real" interpolation theory.

Proposition 1.6. The interpolation norm \|\|$_{\theta, q ; p}$ (or rather, the method of constructing it) is exact of exponent $\theta$.

Proof. Let $T$ be a linear operator from $V$ to $W$ with $\|\quad\|_{0, W}$ and $\|\quad\|_{1, W}$ satisfying (2). We estimate

$$
\begin{aligned}
K_{p}(t, T v) & =\inf \left\{\left(\left\|w_{0}\right\|_{0, W}^{p}+t^{p}\left\|w_{1}\right\|_{1, W}^{p}\right)^{1 / p} ; \quad T v=w_{0}+w_{1}\right\} \\
& \leqq \inf \left\{\left(\left\|T v_{0}\right\|_{0}^{p}, W+\left\|T v_{1}\right\|_{1}^{p}, W\right)^{1 / p}: \quad v=v_{0}+v_{1}\right\} \\
& \leqq \inf \left\{\left(M_{0}^{p}\left\|v_{0}\right\|_{0}^{p}+t^{p} M_{1}^{p}\left\|v_{1}\right\|_{1}^{p}\right)^{1 / p} ; \quad v=v_{0}+v_{1}\right\} \\
& \leqq M_{0} \inf \left\{\left(\left\|v_{0}\right\|_{0}^{p}+\left(t M_{1} / M_{0}\right)^{p}\left\|v_{1}\right\|_{1}^{p}\right)^{1 / p} ; \quad v=v_{0}+v_{1}\right\} \\
& =M_{0} K_{p}\left(t M_{1} / M_{0}, v\right) .
\end{aligned}
$$

We denote the corresponding \|\|$_{\theta, q ; p}$-norm on $W$ (arising from \|\|$_{0, W},\|\|_{1, W}$ ) by \|\|$_{\theta, q ; p, W}$. Then we have

$$
\|T v\|_{\theta, q ; p, W}=\left[\int_{0}^{\infty}\left\{t^{-\theta} K_{p}(t, T v)\right\}^{q} d t / t\right]^{1 / q}
$$




$$
\begin{aligned}
& \leqq M_{0}\left[\int_{0}^{\infty}\left\{t^{-\theta} K_{p}\left(t M_{1} / M_{0}, v\right)\right\}^{q} d t / t\right]^{1 / q} \\
& =M_{0}\left(M_{0} / M_{1}\right)^{-\theta}\left[\int_{0}^{\infty}\left\{t^{-\theta} K_{p}(t, v)\right\}^{q} d t / t\right]^{1 / q} \\
& =M_{0}^{1-\theta} M_{1}^{\theta}\|v\|_{\theta, q ; p} .
\end{aligned}
$$

Here, in the third line we changed variables from $t M_{1} / M_{0}$ to $t$.

In what follows, we will mainly consider $K_{2}(t, \cdot)$, which is suitable to describe quadratic norms.

Definition 1.7. For each $0<\theta<1$, we set

$$
\begin{aligned}
\|v\|_{\theta, 2 ; 2} & =\left[\int_{0}^{\infty}\left\{t^{-\theta} K_{2}(t, v)\right\}^{2} d t / t\right]^{1 / 2}, \\
\|\quad\|_{\theta, 2,2} & =F_{\theta}\left(\begin{array}{lll}
\| & \left\|_{0},\right\| \|_{1}
\end{array}\right)
\end{aligned}
$$

so that $F_{\theta}$ is an exact interpolation method of exponent $\theta$.

Theorem 1.8. (WYDL concavity for $F_{\theta}$ ). Let $\|\quad\|_{i}, i=1,2,3,4,5,6$, be six norms on $V$ and $\alpha, \beta \geqq 0,0<\theta<1$. If $\alpha\|\quad\|_{1}^{2}+\beta\|\quad\|_{2}^{2} \leqq\|\quad\|_{3}^{2}, \alpha\|\quad\|_{4}^{2}+\beta\|\quad\|{ }_{5}^{2} \leqq\|\|_{6}^{2}$, then we have $\alpha\|\quad\|_{7}^{2}+\beta\|\quad\|_{8}^{2} \leqq\|\|_{9}^{2}$. Here, the three norms \|\|$_{7},\|\|_{8},\|\|_{9}$ on $V$ are $F_{\theta}\left(\|\|_{1},\|\|_{4}\right), F_{\theta}\left(\|\|_{2},\|\|_{5}\right), F_{\theta}\left(\|\|_{3},\|\|_{6}\right)$ respectively.

Proof. We compute $\|v\|_{7}^{2}$ as follows:

$$
\begin{aligned}
\|v\|_{7}^{2} & =\int_{0}^{\infty}\left\{t^{-\theta} K_{2}(t, v)\right\}^{2} d t / t \\
& =\int_{0}^{\infty} \inf \left\{\left\|v_{0}\right\|_{1}^{2}+t^{2}\left\|v_{1}\right\|_{4}^{2} ; \quad v=v_{0}+v_{1}\right\} t^{-2 \theta-1} d t,
\end{aligned}
$$

and similar expressions hold for $\|v\|_{8}^{2}$ and $\|v\|_{9}^{2}$. Thus, it is sufficient to show:

$$
\begin{aligned}
& \alpha \inf \left\{\left\|v_{0}\right\|_{1}^{2}+t^{2}\left\|v_{1}\right\|_{4}^{2} ; \quad v=v_{0}+v_{1}\right\} \\
& +\beta \inf \left\{\left\|v_{0}\right\|_{2}^{2}+t^{2}\left\|v_{1}\right\|_{5}^{2} ; \quad v=v_{0}+v_{1}\right\} \\
& \leqq \inf \left\{\left\|v_{0}\right\|_{3}^{2}+t^{2}\left\|v_{1}\right\|_{6}^{2} ; \quad v=v_{0}+v_{1}\right\} .
\end{aligned}
$$

However, this follows immediately from $\alpha\|\|_{1}^{2}+\beta\|\quad\|_{2}^{2} \leqq\|\quad\|_{3}^{2}$ and $\alpha\|\|_{4}^{2}+$ $\beta\|\quad\|_{5}^{2} \leqq\|\quad\|_{6}^{2}$.

Remark 1.9. The reader might observe that the above proof is so simple because: (i) (4) is obvious due to variational expressions of the three involved quantities, (ii) each norm is just an integral of the corresponding term in (4). The above simple arguments based on (i) and (ii) will be repeatedly used. 
Remark 1.10. In [30], Uhlmann proved a similar result for his interpolation method $Q I_{\theta}$. His $Q I$ has just a uniqueness theorem (not an existence result except when norms are quadratic), while our $F_{\theta}$ is constructive. Later we will show that $F_{\theta}$ and $Q I_{\theta}$ are the same (up to a scalar multiple) for quadratic norms so that $Q I_{\theta}$ is exact of exponent $\theta$.

\section{Quadratic Interpolation, Unbounded Operator Approach}

In this section and Sect. 3, we consider a quadratic interpolation method, that is, an interpolation method giving rise to a new quadratic norm from a pair of such norms. As a principle, the approach in Sect. 3 (bounded operator approach) is enough. We however deal with a certain unbounded operator here, which will prove more suitable for applications in Sects. 4 and 5.

We begin with the following main technical tool:

Lemma 2.1. Let $h$ be a positive self-adjoint operator on a Hilbert space $K$ with a form core $D$. Then, for each $\xi \in D$ and $t>0$, we have

$$
\left(h(t+h)^{-1} \xi \mid \xi\right)=\inf \{(h \zeta \mid \zeta) / t+(\eta \mid \eta) ; \quad \xi=\zeta+\eta, \quad \zeta, \eta \in D\} .
$$

Here, $(h \zeta \zeta)$ should be understood as a form $([18])$.

Proof. Due to $(h / t)(1+(h / t))^{-1}=h(t+h)^{-1}$, we may and do assume that $t=1$ in what follows. Also, by making use of the spectral decomposition theorem, it is easy to check:

$$
\left\|(1+h)^{1 / 2} \zeta\right\| \leqq\left\|(2+h)^{1 / 2} \zeta\right\| \leqq \sqrt{2}\left\|(1+h)^{1 / 2} \zeta\right\|, \quad \zeta \in K
$$

Thus, the graph norms of $h^{1 / 2}$ and $(1+h)^{1 / 2}$ are equivalent so that $D$ is an (operator) core for $(1+h)^{1 / 2}$.

When $\xi=\zeta+\eta(\zeta, \eta \in D)$, it is straightforward to show:

$$
\begin{aligned}
(h \zeta \mid \zeta)+(\eta \mid \eta) & =\left(h(1+h)^{-1} \xi \mid \xi\right)+\left\|(1+h)^{1 / 2}\left(h(1+h)^{-1} \xi-\eta\right)\right\|^{2} \\
& \geqq\left(h(1+h)^{-1} \xi \mid \xi\right) .
\end{aligned}
$$

We now note that (although $h(1+h)^{-1} \xi$ may or may not belong to $\left.D\right) h(1+h)^{-1} \xi$ belongs to the domain of $h^{1 / 2}$, or equivalently, that of $(1+h)^{1 / 2}$. Since $D$ is a core for $(1+h)^{1 / 2}$, the second term of the above right hand side can be arbitrarily small by an appropriate choice of $\eta \in D$. This completes the proof.

(Q.E.D.)

In the rest of the section, we will consider a vector space $V$ equipped with compatible (see Remark 2.3) quadratic norms \|\|$_{\alpha}$ and \|\|$_{\beta}$, and quadratic interpolation methods. Namely, \|\|$_{\alpha}$ and \|\|$_{\beta}$ arise from sesquilinear forms $\alpha, \beta$ on $V$ :

$$
\|v\|_{\alpha}^{2}=\alpha(v, v), \quad\|v\|_{\beta}^{2}=\beta(v, v), \quad v \in V .
$$

Let $K$ be the completion of $V$ under $\|\quad\|_{\alpha}$ and we regard $V$ as a (dense) subspace of the Hilbert space $K$ whose inner product is denoted by $(\cdot \mid \cdot)(=\alpha(\cdot, \cdot))$ on $V$ ). Hence $\beta$ 
is a sesquilinear form on $K$ whose domain is dense. The compatibility between \|\|$_{\alpha}$ and \|\|$_{\beta}$ forces that $\beta$ is closable (as a form, see [18]). Let $h$ be the positive selfadjoint operator on $K$ corresponding to the closure of $\beta$ ([18]) so that we have

$$
\begin{gathered}
\beta\left(v_{1}, v_{2}\right)=\left(h^{1 / 2} v_{1} \mid h^{1 / 2} v_{2}\right), \quad v_{i} \in V \\
\left(=\left(h v_{1} \mid v_{2}\right) \text { in a form sense }\right) .
\end{gathered}
$$

From the construction, $V$ is automatically an (operator) core for $h^{1 / 2}$.

We can express \|\|$_{\theta, 2 ; 2}\left(=F_{\theta}\left(\|\|_{\alpha},\|\|_{\beta}\right)\right.$, Definition 1.7) in terms of the operator $h$. For the sake of completeness, we give the proof of the following result (see p. 98, [23]):

Theorem 2.2. For each $v \in V$ and $0<\theta<1$, we have

$$
\|v\|_{\theta, 2 ; 2}=(\pi / 2 \sin (\pi \theta))^{1 / 2}\left\|h^{\theta / 2} v\right\|, \quad v \in V .
$$

Proof. Since $\|v\|_{\beta}^{2}=(h v \mid v)$, we have

$$
\begin{aligned}
K_{2}(t, v)^{2} & =\inf \left\{\left(v_{0} \mid v_{0}\right)+t^{2}\left(h v_{1} \mid v_{1}\right) ; \quad v=v_{0}+v_{1}, \quad v_{i} \in V\right\} \\
& =\left(h\left(t^{-2}+h\right)^{-1} v \mid v\right) . \quad(\text { Lemma 2.1) }
\end{aligned}
$$

Let $h=\int_{0}^{\infty} \lambda d e_{\lambda}$ be the spectral decomposition of $h$. Fubini's theorem (for positive functions) yields:

$$
\begin{aligned}
\|v\|_{\theta, 2 ; 2}^{2} & =\int_{0}^{\infty}\left(\int_{0}^{\infty} \lambda\left(t^{-2}+\lambda\right)^{-1} d\left\|e_{\lambda} v\right\|^{2}\right) t^{-2 \theta-1} d t \\
& =\int_{0}^{\infty} \lambda\left(\int_{0}^{\infty} t^{-2 \theta-1}\left(t^{-2}+\lambda\right)^{-1} d t\right) d\left\|e_{\lambda} v\right\|^{2} .
\end{aligned}
$$

By direct computations (or see the table of Mellin transforms), we have

$$
\begin{aligned}
\|v\|_{\theta, 2 ; 2}^{2} & =\int_{0}^{\infty} \lambda\left(\pi \lambda^{\theta-1} / 2 \sin (\pi \theta)\right) d\left\|e_{\lambda} v\right\|^{2} \\
& =(\pi / 2 \sin (\pi \theta)) \int_{0}^{\infty} \lambda^{\theta} d\left\|e_{\lambda} v\right\|^{2} \\
& =(\pi / 2 \sin (\pi \theta))\left\|h^{\theta / 2} v\right\|^{2} .
\end{aligned}
$$

Remark 2.3. So far, we have been assuming compatibility of two norms. From now on, this is not necessary except in Theorem 3.4. Indeed, all results will be proved as just consequences of variational expressions, and compatibility plays no role. (This is also the case in Theorem 1.8.)

\section{Quadratic Interpolation, Bounded Operator Approach}

In this section, we still restrict ourselves to quadratic norms. We will not use unbounded operators in this section.

As before, we consider a vector space $V$ equipped with two quadratic (semi-) 
norms \|\|$_{\alpha},\|\|_{\beta}$. The following definition is inspired by Pusz-Woronowicz, [24]:

Definition 3.1. We say that $\left(V,\|\|_{\alpha},\|\|_{\beta}\right)$ is represented by a quadruple $(K, i, a, b)$ if $i$ is a linear mapping from $V$ onto a dense subspace in a Hilbert space $K$, and commuting positive operators $a$ and $b$ on $K$ satisfy

$$
\alpha\left(v_{1}, v_{2}\right)=\left(\operatorname{ai}\left(v_{1}\right) \mid i\left(v_{2}\right)\right), \quad \beta\left(v_{1}, v_{2}\right)=\left(b i\left(v_{1}\right) \mid i\left(v_{2}\right)\right), \quad v_{i} \in V .
$$

There exists the canonical construction of a representation from a given $\left(V,\|\|_{\alpha},\|\|_{\beta}\right)$ as follows: Let $K$ be the completion of $V(V /\{v \in V ;\|v\|=0\}$ if $\alpha, \beta$ are seminorms) under the quadratic norm $\|v\|=\left(\|v\|_{\alpha}^{2}+\|v\|_{\beta}^{2}\right)^{1 / 2}$, and $i: V \rightarrow K$ be the natural inclusion. Since \|\|$_{\alpha} \leqq\|\|$, Riesz' theorem guarantees that there is a bounded positive operator $k$ on $K$ satisfying $\alpha\left(v_{1}, v_{2}\right)=\left(k i\left(v_{1}\right) \mid i\left(v_{2}\right)\right), v_{i} \in V$. Clearly, $(K, i, k, 1-k)$ represents $\left(V,\|\|_{\alpha},\|\|_{\beta}\right)$.

Although we will deal with $a$ representation, the notation $(K, i, k, 1-k)$ will be reserved for the canonical representation in what follows.

(The first part of) the next result is closely related to our Lemma 2.1, and was independently obtained by several authors, $[1-4,24]$.

Lemma 3.2. Let $a, b$ be commuting positive operators on a Hilbert space $K$, and $D$ be a dense subspace in $K$. For each $\xi \in K$, we have

$$
\left.\left(a b(a+b)^{-1} \xi \mid \xi\right)=\inf \{a \zeta \mid \zeta)+(b \eta \mid \eta) ; \quad \xi=\zeta+\eta, \quad \zeta, \eta \in D\right\} .
$$

Thus, when $\left(V,\|\|_{\alpha},\|\|_{\beta}\right)$ is represented by $(K, i, a, b)$, we have

$$
\left(a b(a+b)^{-1} i(v) \mid i(v)\right)=\inf \left\{\left\|v_{1}\right\|_{\alpha}^{2}+\left\|v_{2}\right\|_{\beta}^{2} ; \quad v=v_{1}+v_{2}\right\} .
$$

Actually, if one replaces $(h, 1)$ by $(a, b)$ in the proof of Lemma 2.1, almost same arguments there yield this lemma. Furthermore, the result remains valid for any $a, b \geqq 0([a, b] \neq 0)$ under a certain interpretation of $a b(a+b)^{-1}$ (for example, $\left(a^{-1}+b^{-1}\right)^{-1}$ if all inverses make sense). Or rather some authors consider the expression in the above lemma as the definition of the operator in the left hand side. In the literature, this is called either the parallel sum or the (half of the) harmonic mean (of $a$ and $b$ ). An interesting interpretation of a parallel sum (in terms of theory of electric circuits) can be found in [1].

We may interpret Lemma 2.1 as follows: If $\left(V,\|\|_{\alpha},\|\|_{\beta}\right)$ is "unboundedly" represented by (the completion of $V$ under \|\|$_{\alpha}$, the natural inclusion map, $1, h$ ) as in Sect. 2 with the "correct" core condition (as stated in Lemma 2.1), then Lemma 3.2 remains valid. However, existence of such an unbounded representation is guaranteed only when two norms in question are compatible. (see Remark 2.3.)

If $(K, i, k, 1-k)$ is the canonical representation of $\left(V,\|\|_{\alpha},\|\|_{\beta}\right)$, then, for $0<s$ $<1, a=s k$ and $b=(1-s)(1-k)$ commute. Thus, Lemma 3.2 implies:

Corollary 3.3. For each $0<s<1, v \in V$, we have

$$
\begin{aligned}
\left(k(1-k)\{s k+(1-s)(1-k)\}^{-1} i(v) \mid i(v)\right)= & s^{-1}(1-s)^{-1} \inf \left\{s\left\|v_{1}\right\|_{\alpha}^{2}\right. \\
& \left.+(1-s)\left\|v_{2}\right\|_{\beta}^{2} ; v=v_{1}+v_{2}\right\} .
\end{aligned}
$$


We now try to improve Theorem 1.8 (by restricting ourselves to quadratic interpolation methods). We will use the complete characterization of all exact quadratic interpolation methods obtained by Donoghue Jr., [9], and Lions-Foias, [12]. It is worth pointing out that in both [9] and [12] Löwner's theory on operator monotone functions, [10], is crucially used.

With the canonical representation $(K, i, k, 1-k)$ of a generic $\left(V,\|\|_{\alpha},\|\|_{\beta}\right)$, the main result in [9] states:

Theorem 3.4. The exact quadratic interpolation methods $F$ are in one-to-one correspondence with the set of all functions $f(\lambda)$ of the form:

$$
f(\lambda)=\int_{0}^{1} \lambda(1-\lambda)\{s \lambda+(1-s)(1-\lambda)\}^{-1} d v(s),
$$

$d v(s)$ being a positive Radon measure on the closed unit interval $[0,1]$. Here, \|\|$_{*}=F\left(\|\|_{\alpha},\|\|_{\beta}\right)$ is determined $b y$

$$
\|v\|_{*}=(f(k) i(v) \mid i(v))^{1 / 2}, v \in V .
$$

Before proceeding further, we remark that this theorem remains valid even for quadratic semi-norms under a certain modification. (see Theorem $1^{\prime}$ and $2^{\prime}$ in [9].). The next result shows that all exact quadratic interpolation methods enjoy the WYDL concavity.

Theorem 3.5. (WYDL concavity for an exact quadratic interpolation method). Let $F$ be an exact quadratic interpolation method arising from $f(\lambda)$ as in the previous theorem, and \|\|$_{i}, i=1,2,3,4,5,6$, be quadratic (semi-)norms on a vector space $V$. If $\alpha\|\quad\|_{1}^{2}+\beta\|\quad\|_{2}^{2} \leqq\|\quad\|_{3}^{2}$ and $\alpha\|\quad\|_{4}^{2}+\beta\|\quad\|{ }_{5}^{2} \leqq\|\|_{6}^{2}$, then we have $\alpha\|\quad\|_{7}^{2}+\beta\|\quad\|_{8}^{2} \leqq\|\|_{9}^{2}$, where \|\|$_{7},\|\quad\|_{8},\|\|_{9}$ are $F\left(\|\|_{1},\|\|_{4}\right), F\left(\|\|_{2}\right.$, \|\|$\left._{5}\right), F\left(\|\|_{3},\|\|_{6}\right)$ respectively.

Proof. The definition of \|\|$_{7}$ and Corollary 3.3 imply:

$$
\begin{aligned}
\|v\|_{7}^{2}= & \int_{[0,1]}\left(k(1-k)\{s k+(1-s)(1-k)\}^{-1} i(v) \mid i(v)\right) d v(s) \\
= & \int_{(0,1)}\left(k(1-k)\{s k+(1-s)(1-k)\}^{-1} i(v) \mid i(v)\right) d v(s) \\
& +\gamma\|v\|_{1}^{2}+\delta\|v\|_{4}^{2} \\
= & \int_{(0,1)} s^{-1}(1-s)^{-1} \inf \left\{s\left\|v_{0}\right\|_{1}^{2}+(1-s)\left\|v_{1}\right\|_{4}^{2} ;\right. \\
& \left.v=v_{0}+v_{1}\right\} d v(s)+\gamma\|v\|_{1}^{2}+\delta\|v\|_{4}^{2}
\end{aligned}
$$

with $\gamma=d v(\{0\})$ and $\delta=d v(\{1\})$. We also have the corresponding expressions for $\|v\|_{8}^{2},\|v\|_{9}^{2}$ with the same $d v, \gamma, \delta$. 
By the assumption, we have

$$
\begin{aligned}
& \alpha \inf \left\{s\left\|v_{0}\right\|_{1}^{2}+(1-s)\left\|v_{1}\right\|_{4}^{2} ; v=v_{0}+v_{1}\right\} \\
+ & \beta \inf \left\{s\left\|v_{0}\right\|_{2}^{2}+(1-s)\left\|v_{1}\right\|_{5}^{2} ; v=v_{0}+v_{1}\right\} \\
\leqq & \inf \left\{s\left\|v_{0}\right\|_{3}^{2}+(1-s)\left\|v_{1}\right\|_{6}^{2} ; v=v_{0}+v_{1}\right\} .
\end{aligned}
$$

Thus, the result is obvious from the integral expressions in the first half of the proof.

(Q.E.D.)

Remark 3.6. One can prove (an easier) half of Theorem 3.4 using the above arguments. Consider the quadratic interpolation method of giving

$$
\|v\|_{*, s}=\left(k(1-k)\{s k+(1-s)(1-k)\}^{-1} i(v) \mid i(v)\right)
$$

(from $\|v\|_{\alpha}=(k i(v) \mid i(v))^{1 / 2}$ and $\left.\|v\|_{\beta}=((1-k) i(v) \mid i(v))^{1 / 2}\right)$. As in the first part of the proof of Proposition 1.6, (2) and Corollary 3.3 imply $\|T v\|_{*, s} \leqq \operatorname{Max}\left(M_{0}, M_{1}\right) \times$ $\|v\|_{*, s}$. Thus, passing to the integral in the proof of the preceding theorem, we obtain the desired exactness.

Finally in this section, we identify Uhlmann's quadratic interpolation method $Q I_{\theta}$ with $F_{\theta}$ in Definition 1.7. (See Remark 1.10.) His construction is based on geometric means in [24], and some complex analysis. In our terminology, $Q I_{\theta}\left(\|\|_{\alpha},\|\|_{\beta}\right)$, denoted by \|\|$_{\theta}$ for simplicity in the proof below, is given by

$$
\|v\|_{\theta}=\left(a^{1-\theta} b^{\theta} i(v) \mid i(v)\right)^{1 / 2}, \quad v \in V,
$$

if $\left(V,\|\|_{\alpha},\|\|_{\beta}\right)$ is represented by $(K, i, a, b)$. (See Proposition 10, [30].)

Theorem 3.7. For quadratic norms \|\|$_{\alpha},\|\|_{\beta}$, we have

$$
Q I_{\theta}\left(\|\|_{\alpha},\|\|_{\beta}\right)=(2 \sin (\pi \theta) / \pi)^{1 / 2} F_{\theta}\left(\|\|_{\alpha},\|\|_{\beta}\right), 0<\theta<1 .
$$

Proof. Using the joint spectral measure $d e_{\lambda \mu}$ for commuting $a$, $b$, we directly compute

$$
\begin{aligned}
& \int_{0}^{\infty}\left(t^{2} a b\left\{a+t^{2} b\right\}^{-1} i(v) \mid i(v)\right) t^{-2 \theta-1} d t \\
= & \int_{0}^{\infty} \int_{0}^{\infty} \lambda \mu\left(\int_{0}^{\infty}\left(\lambda+t^{2} \mu\right)^{-1} t^{1-2 \theta} d t\right) d\left\|e_{\lambda \mu} i(v)\right\|^{2} \\
= & \int_{0}^{\infty} \int_{0}^{\infty}(\pi / 2 \sin (\pi \theta)) \lambda^{1-\theta} \mu^{\theta} d\left\|e_{\lambda \mu} i(v)\right\|^{2} \\
= & (\pi / 2 \sin (\pi \theta))\left(a^{1-\theta} b^{\theta} i(v) \mid i(v)\right) \\
= & (\pi / 2 \sin (\pi \theta))\|v\|_{\theta}^{2} .
\end{aligned}
$$

Thus, Lemma 3.1 shows:

$$
(\pi / 2 \sin (\pi \theta))\|v\|_{\theta}^{2}=\int_{0}^{\infty} \inf \left\{\left\|v_{0}\right\|_{\alpha}^{2}+t^{2}\left\|v_{1}\right\|_{\beta}^{2} ; v=v_{0}+v_{1}\right\} t^{-2 \theta-1} d t .
$$


But the right hand side is the square of $F_{\theta}\left(\|\|_{\alpha},\|\|_{\beta}\right)$.

In particular, this result and Proposition 1.6 guarantee that the quadratic interpolation method $Q I_{\theta}$ is exact of exponent $\theta$. However, this result is implicit in [30] (although just exactness is explicitly mentioned, see Proposition 9, [30].). And there is only one exact quadratic interpolation method of exponent $\theta$ up to a scalar multiple. The proof of the uniqueness will be published elsewhere.

\section{F. Hansen's Inequality}

We will give an alternative proof of F. Hansen's inequality, [15]. (see Theorem 4.2) More importantly, we will point out and clarify a close relation between this inequality and quadratic interpolation theory.

The following result is an easy consequence of Löwner's theory ([10]) so that we leave full details to the reader. (Actually, it is proved in p.216, [31].)

Lemma 4.1. Let $g(\lambda)$ be a non-negative operator monotone function on $[0, \infty)$. Then there exist unique non-negative numbers $\varepsilon_{1}, \varepsilon_{2}$ and a unique positive finite measure $d \mu$ on $(0, \infty)$ such that

$$
g(\lambda)=\varepsilon_{1}+\varepsilon_{2} \lambda+\int_{0}^{\infty} \lambda(t+\lambda)^{-1} d \mu(t), \lambda \geqq 0 .
$$

We remark that conversely a function $g(\lambda)$ given by the above integral expression is operator monotone on $[0, \infty)$ due to Lemma 2.1 .

Theorem 4.2. (F. Hansen's inequality, [15]). Let $x, y$ be positive (bounded) operators on a Hilbert space $K$, and a be a contraction. For any non-negative operator monotone function $g(\lambda)$ on $[0, \infty), x \leqq y$ implies $a^{*} g(x) a \leqq g\left(a^{*} y a\right)$. In particular (and equivalently), we always have $a^{*} g(x) a \leqq g\left(a^{*} x a\right)$.

First Proof. We simply compute $(\xi \in K)$ :

$$
\begin{aligned}
\left(a^{*} g(x) a \xi \mid \xi\right)= & (g(x) a \xi \mid a \xi)=\varepsilon_{1}(a \xi \mid a \xi)+\varepsilon_{2}(x a \xi \mid a \xi) \\
& +\int_{0}^{\infty} \inf \left\{\left(x \zeta_{1} \mid \zeta_{1}\right) / t+\left(\zeta_{2} \mid \zeta_{2}\right) ; a \xi=\zeta_{1}+\zeta_{2}\right\} d \mu(t) \\
\leqq & \varepsilon_{1}(a \xi \mid a \xi)+\varepsilon_{2}(x a \xi \mid a \xi) \\
& +\int_{0}^{\infty} \inf \left\{\left(x a \xi_{1} \mid a \xi_{1}\right) / t+\left(a \xi_{2} \mid a \xi_{2}\right) ; \quad \xi=\xi_{1}+\xi_{2}\right\} d \mu(t) \\
\leqq & \varepsilon_{1}(\xi \mid \xi)+\varepsilon_{2}(y a \xi \mid a \xi) \\
& +\int_{0}^{\infty} \inf \left\{\left(a^{*} y a \xi_{1} \mid \xi_{1}\right) / t+\left(\xi_{2} \mid \xi_{2}\right) ; \quad \xi=\xi_{1}+\xi_{2}\right\} d \mu(t) \\
\quad(\text { since } x \leqq y \text { and }\|a\| \leqq 1) & \left(g\left(a^{*} y a\right) \xi \mid \xi\right) .
\end{aligned}
$$


One can also find another proof in [13]. This inequality obviously remains valid for a positive measurable operator in the sense of Segal, [27]. As an application of this fact, a certain dominated convergence theorem for probability gages ([27]) is obtained in [21].

A half of the proof of Theorem 3.4 (see Remark 3.6.) and the above proof of F. Hansen's inequality are based on the same principle (variational expressions). Actually, not only their proofs but also their statements themselves are "identical." The rest of the section will be devoted to explaining this fact.

Remark 4.3. For any non-negative operator monotone function $g(\lambda)$ on $[0, \infty)$, we define an exact quadratic interpolation method $F_{g}$ by

$$
\begin{aligned}
\|\quad\|_{*} & =F_{g}\left(\|\quad\|_{\alpha},\|\quad\|_{\beta}\right) \\
\|v\|_{*} & =(g(h) v \mid v)^{1 / 2}, v \in V,
\end{aligned}
$$

with $(K, h)$ constructed in Sect. 2 from compatible \|\|$_{\alpha},\|\|_{\beta}$. Indeed, the exactness of $F_{g}$ can be immediately observed if one repeats the arguments in Remark 3.6 together with Lemma 2.1 and Lemma 4.1. (Also, \|\|$_{*}$ does not depend on a choice of $(K, h)$ as long as $V$ is a form core for $h$ due to the variational expression, Lemma 2.1.) Actually, all $F_{g}{ }^{\prime} s(g \neq 0)$ exhaust all exact quadratic interpolation methods described in Theorem 3.4. Since we do not need this fact, we will postpone a proof elsewhere.

We now "reprove" Theorem 4.2 to explain the fact that $F$. Hansen's inequality follows from quadratic interpolation theory.

Second Proof of Theorem 4.2. Let $\|\cdot\|=(\cdot \mid \cdot)^{1 / 2}$ be the Hilbert space norm of $K$, and we set $h_{1}=a^{*} y a, h_{2}=x$. We now consider the two pairs of quadratic semi-norms $\left(\|\cdot\|,\left(h_{1} \cdot \mid \cdot\right)^{1 / 2}\right)$ and $\left(\|\cdot\|,\left(h_{2} \cdot \cdot \cdot\right)^{1 / 2}\right)$ on $K$ (which are represented by $h_{1}$ and $h_{2}$ respectively in the sense of sect. 2). A given contraction $a: K \rightarrow K$ satisfies

$$
\|a \xi\| \leqq\|\xi\|,\left(h_{2} a \xi \mid a \xi\right)=\left(h_{1} \xi \mid \xi\right), \xi \in K .
$$

Since $F_{g}$ is exact (Remark 4.3), we must have

$$
\left(g\left(h_{2}\right) a \xi \mid a \xi\right)^{1 / 2} \leqq\left(g\left(h_{1}\right) \xi \mid \xi\right)^{1 / 2},
$$

which is same as $a^{*} g(x) a \leqq g\left(a^{*} y a\right)$.

On the other hand, $a^{*} g(x) a \leqq g\left(a^{*} x a\right)$ for any $x \geqq 0,\|a\| \leqq 1$, if and only if $g(\lambda)$ is operator monotone as shown in [16]. Thus, the validity of $F$. Hansen's inequality implies the exactness of $F_{g}$ (Remark 4.3).

\section{WYDL Concavity in the Context of von Neumann Algebras}

We will obtain a certain generalization of Araki's version, [6], of WYDL concavity (in the context of von Neumann algebras). As one can easily imagine, all results in this section can be regarded as consequences of our most general result Theorem 3.5 (see Remark 5.5). However, we will provide direct proofs because they are equally simple as reductions from Theorem 3.5.

To express the WYDL concavity for a von Neumann algebra, which does not 
admit a trace, we need the notion of a standard form. In the rest of the section, let $M$ be a ( $\sigma$-finite) von Neumann algebra with a standard form $(M, H, J, P$ দ $)$ in the sense of $[6,14]$. Hence, $M$ is standardly acting on a Hilbert space $H$, and a natural cone $P$ is a self-dual cone in $H$ such that the map; $\xi \in P \natural \rightarrow \omega_{\xi} \in M_{*}^{+}$is bijective. A unitary involution $J$ on $H$ satisfies $J M J=M^{\prime}$, and $J \xi=\xi$ for each $\xi \in \mathrm{P}$. For each $\phi \in M_{*}^{+}$, we denote its unique implementing vector in $P$ घ by $\xi_{\phi}$. For $\phi, \psi$ in $M_{*}^{+}$( $\phi$ is faithful for simplicity), a (unique) positive self-adjoint operator $\Delta_{\psi \phi}$ on $H$ such that $J \Delta_{\psi \phi}^{1 / 2} x \xi_{\phi}=x^{*} \xi_{\psi}, x \in M$, and $M \xi_{\phi}$ is a form core is known as the relative modular operator (of $\psi$ relative to $\phi$ ). (See [6] or [19] for example.) later.

The following example might be intuitive for non-specialists, and will be used

Example 5.1. Let $K$ be a (finite or infinite dimensional) Hilbert space, and $C_{2}(K)$ (respectively $\left.C_{2}^{+}(K)\right)$ is the Hilbert space of all Hilbert-Schmidt class operators on $K$ (respectively the cone consisting of all positive Hilbert-Schmidt class operators on $K)$. Then $\left(B(K), C_{2}(K), J={ }^{*}, C_{2}^{+}(K)\right)$ is a standard form, where the von Neumann algebra $B(K)$ should be understood to act on $C_{2}(K)$ as left multiplications. The predual $B(K)_{*}$ is identified with $C_{1}(K)$, the set of all trace class operators, via $\phi \in B(K)_{*}^{+} \rightarrow h_{\phi} \in C_{1}^{+}(K)$ with $\phi=\operatorname{Tr}\left(h_{\phi} \cdot\right)$. Then the unique implementing "vector" $\xi_{\phi}$ in $C_{2}^{+}(K)$ is $h_{\phi}^{1 / 2}$ due to $\phi(x)=\operatorname{Tr}\left(h_{\phi} x\right)=\operatorname{Tr}\left(x h_{\phi}^{1 / 2} h_{\phi}^{1 / 2}\right)=\left(x h_{\phi}^{1 / 2} \mid h_{\phi}^{1 / 2}\right)$. We also remark that, in the present context, the relative modular operator $\Delta_{\psi \phi}$ on $C_{2}(K)$ is given by

$$
\Delta_{\psi \phi}^{\theta / 2} x \xi_{\phi}=\Delta_{\psi \phi}^{\theta / 2}\left(x h_{\phi}^{1 / 2}\right)=h_{\psi}^{\theta / 2} x h_{\phi}^{1 / 2-\theta / 2}, x \in B(K), 0 \leqq \theta \leqq 1 .
$$

Thus, we obtain

$$
\begin{aligned}
\left\|\Delta_{\psi \phi}^{\theta / 2} x \xi_{\phi}\right\|^{2} & =\operatorname{Tr}\left(\left(h_{\psi}^{\theta / 2} x h_{\phi}^{1 / 2-\theta / 2}\right)^{*}\left(h_{\psi}^{\theta / 2} x h_{\phi}^{1 / 2-\theta / 2}\right)\right) \\
& =\operatorname{Tr}\left(h_{\phi}^{1-\theta} x^{*} h_{\psi}^{\theta} x\right)
\end{aligned}
$$

which is a typical quantity appearing in the WYDL concavity in the context of the matrix algebra (see the very first part of Sect. 0).

Returning to a general von Neumann algebra, we state our main result in the section.

Theorem 5.2. (WYDL concavity). Let $g(\lambda)$ be a non-negative operator monotone function on $[0, \infty)$, and $\phi_{1}, \phi_{2}, \phi, \psi_{1}, \psi_{2}, \psi$ be elements in $M_{*}^{+}$. If we have

$$
\alpha \phi_{1}+\beta \phi_{2} \leqq \phi \text { and } \alpha \psi_{1}+\beta \psi_{2} \leqq \psi ; \alpha, \beta \geqq 0,
$$

then, for each $x \in M$, we have

$$
\begin{gathered}
\alpha\left(g\left(\Delta_{\psi_{1} \phi_{2}}\right) x \xi_{\phi_{1}} \mid x \xi_{\phi_{1}}\right)+\beta\left(g\left(\Delta_{\psi_{2} \phi_{2}}\right) x \xi_{\phi_{2}} \mid x \xi_{\phi_{2}}\right) \\
\leqq\left(g\left(\Delta_{\psi \phi}\right) x \xi_{\phi} \mid x \xi_{\phi}\right) .
\end{gathered}
$$

Before going to its proof, we remark that all terms in (6) make sense as forms. Indeed, the function $g(\lambda)$ being concave in the usual sense (due to Lemma 4.1), we have $g(\lambda) \leqq c \lambda+d, \lambda \geqq 0$, for some $c, d \geqq 0$. 
Proof. Due to Lemma 4.1, we have

$$
\begin{aligned}
\left(g\left(\Delta_{\psi \phi}\right) x \xi_{\phi} \mid x \xi_{\phi}\right)= & \varepsilon_{1}\left(x \xi_{\phi} \mid x \xi_{\phi}\right)+\varepsilon_{2}\left(\Delta_{\psi \phi} x \xi_{\phi} \mid x \xi_{\phi}\right) \\
& +\int_{0}^{\infty}\left(\Delta_{\psi \phi}\left(t+\Delta_{\psi \phi}\right)^{-1} x \xi_{\phi} \mid x \xi_{\phi}\right) d \mu(t) .
\end{aligned}
$$

We note that

$$
\begin{aligned}
\left(y \xi_{\phi} \mid y \xi_{\phi}\right) & =\phi\left(y^{*} y\right), \\
\left(\Delta_{\psi \phi} y \xi_{\phi} \mid y \xi_{\phi}\right) & =\left\|\Delta_{\psi \phi}^{1 / 2} y \xi_{\phi}\right\|^{2}=\left\|J y^{*} \xi_{\psi}\right\|^{2} \\
& =\left\|y^{*} \xi_{\psi}\right\|^{2}=\psi\left(y y^{*}\right) .
\end{aligned}
$$

Since $M \xi_{\phi}$ is an (operator) core for $\Delta_{\psi \phi}^{1 / 2}$, Lemma 2.1 (applied to (7)) yields:

$$
\begin{aligned}
\left(g\left(\Delta_{\psi \phi}\right) x \xi_{\phi} \mid x \xi_{\phi}\right)= & \varepsilon_{1} \phi\left(x^{*} x\right)+\varepsilon_{2} \psi\left(x x^{*}\right) \\
& +\int_{0}^{\infty} \inf \left\{\psi\left(y y^{*}\right) / t+\phi\left(z^{*} z\right) ; x=y+z\right] d \mu(t),
\end{aligned}
$$

for generic $\phi, \psi, x$. Thus, as before, (6) follows from the following obvious consequence of (5):

$$
\begin{aligned}
& \alpha \inf \left\{\psi_{1}\left(y y^{*}\right) / t+\phi_{1}\left(z^{*} z\right) ; x=y+z\right\} \\
& +\beta \inf \left\{\psi_{2}\left(y y^{*}\right) / t+\phi_{2}\left(z^{*} z\right) ; x=y+z\right\} \\
& \quad \leqq \inf \left\{\psi\left(y y^{*}\right) / t+\phi\left(z^{*} z\right) ; x=y+z\right\} .
\end{aligned}
$$

The function $g(\lambda)=\lambda^{\theta} \geqq 0,0 \leqq \theta \leqq 1$, being operator monotone on $[0, \infty)$, we have:

Corollary 5.3. ([6]). For each $0 \leqq \theta \leqq 1$ and $x \in M$, (5) in Theorem 5.2 implies

$$
\alpha\left\|\Delta_{\psi_{1} \phi_{1}}^{\theta / 2} x \xi_{\phi_{1}}\right\|^{2}+\beta\left\|\Delta_{\psi_{2} \phi_{2}}^{\theta / 2} \xi_{\phi_{2}}\right\|^{2} \leqq\left\|\Delta_{\psi \phi}^{\theta / 2} x \xi_{\phi}\right\|^{2} .
$$

What is involved here is the following well-known identity (used in theory of semi-groups):

$$
\lambda^{\theta}=(\sin (\pi \theta) / \pi) \int_{0}^{\infty} \lambda(t+\lambda)^{-1} t^{\theta-1} d t
$$

This identity was also used in [11] (in a different way) to obtain an alternative proof of:

Corollary 5.4. ([22]). Let $x$ be a (bounded) operator of a Hilbert space $K$, and $C_{1}^{+}(K)$ be the set of all positive trace class operators on $K$. If $\alpha h_{1}+\beta h_{2} \leqq h$ and $\alpha k_{1}+\beta k_{2} \leqq k\left(h_{1}, h_{2}, h, k_{1}, k_{2}, k \in C_{1}^{+}(K) ; \alpha, \beta \leqq 0\right)$, then we have

$$
\alpha \operatorname{Tr}\left(h_{1}^{1-\theta} x^{*} k_{1}^{\theta} x\right)+\beta \operatorname{Tr}\left(h_{2}^{1-\theta} x^{*} k_{2}^{\theta} x\right) \leqq \operatorname{Tr}\left(h^{1-\theta} x^{*} k^{\theta} x\right),
$$

for each $0 \leqq \theta \leqq 1$. 
Proof. When $M=B(K)$ as in Example 5.1, Corollary 5.3 reduces to this result.

Remark 5.5. A pair $(\phi, \psi)$ of generic elements in $M_{*}^{+}$induces a pair of quadratic forms (hence, semi-norms) on $M$ as follows:

$$
(x, y) \rightarrow \phi\left(y^{*} x\right), \quad(x, y) \rightarrow \psi\left(x y^{*}\right) .
$$

(See Sect. 3, [24].) In terms of a standard Hilbert space and relative modular operators, they are computed as:

$$
\begin{aligned}
\phi\left(y^{*} x\right) & =\left(x \xi_{\phi} \mid y \xi_{\phi}\right), \\
\psi\left(x y^{*}\right) & =\left(y^{*} \xi_{\psi} \mid x^{*} \xi_{\psi}\right)=\left(J \Delta_{\psi \phi}^{1 / 2} y \xi_{\phi} \mid J \Delta_{\psi \phi}^{1 / 2} x \xi_{\phi}\right) \\
& =\left(\Delta_{\psi \phi}^{1 / 2} x \xi_{\phi} \mid \Delta_{\psi \phi}^{1 / 2} y \xi_{\phi}\right)=\left(\Delta_{\psi \phi} x \xi_{\phi} \mid y \xi_{\phi}\right),
\end{aligned}
$$

so that the two semi-norms are "unboundedly represented" by $\left(H, i_{\phi}, 1, \Delta_{\psi \phi}\right)$ with $i_{\phi}(x)=x \xi_{\phi}, x \in M$. (And, actually, these two semi-norms are compatible due to the closedness of relative modular operators.) Thus, the quadratic interpolation method $F_{g}$ described in Remark 4.3 produces the new quadratic semi-norm:

$$
x \in M \rightarrow\left(g\left(\Delta_{\psi \phi}\right) i_{\phi}(x) \mid i_{\phi}(x)\right)^{1 / 2}=\left(g\left(\Delta_{\psi \phi}\right) x \xi_{\phi} \mid x \xi_{\phi}\right)^{1 / 2},
$$

so that all results in this section are special cases of Theorem 3.5.

Some analysis on the two forms in the above remark (called the right and left forms in the literature, $[24,25,30]$ ) and more applications of the general interpolation theory to von Neumann algebras are found in [19, 20, 29]. Also, more systematic analysis on relations among the subjects appearing in the present article will be carried out in a subsequent paper.

Acknowledgement. The author is indebted to Professor Huzihiro Araki for a critical reading of this article and for useful suggestions.

\section{References}

1. Anderson Jr., W. N., Trapp, G. E.: Shorted operators II. SIAM J. Appl. Math. 28, 60-71 (1975)

2. Anderson Jr., W. N., Morley, D. E., Trapp, G. E.: Characterization of parallel sums. Proc. Natl. Acad. Sci. USA, 76, 599-3601 (1979)

3. Ando, T.: Concavity of certain maps on positive definite matrices and applications to Hadamard products. Linear Alg. Appl. 26, 203-241 (1979)

4. Ando, T., Kubo, F.: Means of positive linear operators. Math. Ann. 246, 205-224 (1980)

5. Araki, H.: Some properties of modular conjugation operator of von Neumann algebras and a noncommutative Radon-Nikodym theorem with a chain rule. Pac. J. Math. 50, 309-354 (1974)

6. Araki, H.: Relative entropy of states of von Neumann algebras I, II. Publ. RIMS, Kyoto Univ. 11, 809-833; (1975/76). 13 (1977), 173-192

7. Aronszajn, N., Gagliardo, E.: Interpolation spaces and interpolation method. Ann. Math. Pure Appl. 68, 51-118 (1965)

8. Berg, J., Löfström, J.: Interpolation spaces, an introduction. Berlin Heidelberg, New York : Springer 1976

9. Donoghue Jr., W.: The interpolation of quadratic norms. Acta Math. 148, 251-270 (1967)

10. Donoghue Jr., W.: Monotone matrix functions and analytic continuation. Berlin, Heidelberg, New York: Springer 1974 
11. Epstein, H.: Remarks on two theorems of E. Lieb. Commun. Math. Phys. 31, 317-325 (1973)

12. Foias, C., Lions, J. L.: Sur certains théoréme d'interpolation. Acta Sci. Math. (Szeged) 22, 269-282 (1961)

13. Fujii, J.: Initial conditions on operator monotone functions. Math. Jpn 24, 244-262 (1979)

14. Haagerup, U.: The standard form of von Neumann algebras. Math. Scand. 37, 271-283 (1975)

15. Hansen, F. An operator inequality. Math. Ann. 246, 249-250 (1980)

16. Hansen, F. Pedersen, G.: Jesen's inequality for operators and Löwner's theorem. Preprint

17. Holmstedt, T., Peetre, J.: On certain functions arising in the theory of interpolation spaces. J. Funct. Anal. 4, 88-94 (1969)

18. Kato, T.: Perturbation theory for linear operators. Berlin, Heidelberg, Springer 1966

19. Kosaki, H.: Applications of the complex interpolation method to a von Neumann algebra (Noncommutative $L^{p}$-spaces). Preprint

20. Kosaki, H.: Non-commutative Lorentz spaces associated with a semi-finite von Neumann algebra and applications. Proc. Jpn. Acad. 57, Ser. A, 303-306 (1981)

21. Kosaki, H.: Applications of uniform convexity of non-commutative $L^{p}$-spaces. Preprint

22. Lieb, E.: Convex trace functions and the Wigner-Yanase-Dyson conjecture. Math. 11, 267-288 (1973)

23. Lions, J. L., Magenes, E.: Non-homogeneous boundary value problems. Berlin, Heidelberg, New York: Springer 1972

24. Pusz, W., Woronowitz, S.: Functional calculus for sesquilinear forms and the purification map. Rep. Math. Phys. 5, 159-170 (1975)

25. Pusz, W. Woronowitz, S.: Form convex functions and the WYDL and other inequalities. Lett. Math. Phys. 2, 505-512 (1978)

26. Reed, M., Simon, B.: Methods of modern mathematical physics. New York, San Francisco, London: Academic Press. 1975

27. Segal, I.: A non-commutative extension of abstract integration. Ann. Math. 57, 401-457 (1953)

28. Simon, B.: Trace ideals and their applications. London,. New York, Melbourne: Cambridge University Press. 1979

29. Terp, M.: Interpolation spaces between a von Neumann algebra and its predual. Preprint

30. Uhlmann, A.: Relative entropy and the Wigner-Yanase-Dyson-Lieb concavity in an interpolation theory. Commun. Math. Phys. 54, 21-32 (1977)

Communicated by $\mathrm{H}$. Araki

Received April 1, 1982 
